\title{
TINA Service Management Principle
}

- In Search of A Paradigm Beyond TMN -

\author{
H. Kamata T. Hamada \\ OKI Electric Fujitsu Ltd.
}

TINA-C clo Bellcore, NVC-1C234, 331 Newman Springs Rd., Red Bank, NJ 07701, U.S.A., ph: +1 908758 2715, fax: +1 908758 2865, e-mail: hamada@tinac.com

Keywords: TMN, Management Context, Service Transaction

TMN [1][2] takes a resource-oriented, bottom-up approach, upon which the service and the business aspects come last. TINA service management [3] takes the opposite direction towards TMN. TINA service management is a mapping of FCAPS management requirements onto its resource layer.

The TINA service layer passes its management requirements in the form of Management Contexts (MgmtCtxt), which are carried by a construct called Service Transaction. MgmtCtxts are subsequently interpreted, and the results are then mapped onto the TINA resource layer. Although the TINA resource layer can be built on a monolithic object-oriented framework known as DPE, it is still fully interoperable with TMN, allowing itself to interact with Managed Objects (MO) in various layers of TMN. TINA service is session-oriented, and a set of FCAPS MgmtCtxts are bound to each service session. With the service transaction carrying MgmtCtxts, a consistent service management throughout a distributed,

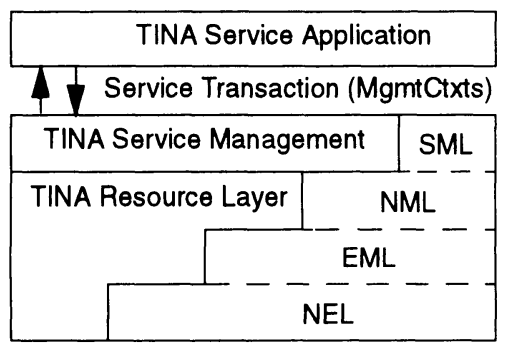

TMN heterogenous environment is guaranteed. Execution of a service-transaction (ex. accounting management) consists of the following three phases.

1. Set-up Phase: MgmtCtxts are interpreted, and necessary resources such as accounting-log record, event-channels are reserved

2. Execution Phase: Being allowed to access authorized data/information sources, the TINA service session is executed. Following the Accounting MgmtCtxt, accounting events may be logged or reported.

3. Wrap-up Phase: Reports from performance management or fault management can be summarized. If the QoS level was satisfactory, the service transaction concludes. Otherwise recovery actions such as charging compensation may be taken.

\section{References}

[1] Principles for a Telecommunication Management Network, ITU-T Recommendation M.3010, 1992.

[2] Generic Requirements for Operations Based on the Telecommunications Management Network (TMN) Architecture, Bellcore, GR-2869-CORE, Oct. 1995.

[3] Service Architecture Ver.4.0, TINA-C, Dec. 1996. 